\title{
Type B Tylosis
}

National Cancer Institute

\section{Source}

National Cancer Institute. Type B Tylosis. NCI Thesaurus. Code C122926.

An inherited form of tylosis that presents by one year and is characterized by benign palmoplantar keratoderma. 